\title{
Crystallographic Processing of Scanning Probe Microscopy Images on the Example of 2D Periodic Molecular Monolayer Arrays
}

\author{
P. Moeck,* J. Straton,* B. Moon Jr., * P. Plachinda,* M. Hietschold, ** H. Glowatzki, *** N. \\ Koch, ${ }^{* * *}$ and J.P. Rabe*** \\ * Department of Physics, Portland State University, PO Box 751, Portland, OR 97207-0751 \\ ** Institute of Physics, Chemnitz University of Technology, Reichenhainer Straße 70, D-09126 \\ Chemnitz, FRG \\ *** Department of Physics, Humboldt University Berlin, Newtonstraße 15, D-12489 Berlin, FRG
}

The defining features of a traditional scanning probe microscope (SPM) are a very fine "probe" that is scanned in two dimensions (2D), in very fine steps, very close to the surface of a sample, and a "probe-sample interactions signal" that is recorded at each scanning increment. This signal may then be digitized and displayed as a function of the magnified scanning steps. The result is a 2D-image of the probe-sample interactions. Just like any other image, a SPM image can be subjected to image processing routines in order to quantify the information that it contains.

Following the common practice of image-based structural electron crystallography [1,2], SPM images can be processed crystallographically in order to quantify their deviations from the 17 plane (i.e. 2D space symmetry) groups. This information can in turn be used to remove from the SPM image all kinds of geometric distortions that are due to the "less than perfect" imaging process.

The combined effects of these distortions result in a point spread function that gives a quantitative measure of the microscope's performance for a certain set of experimental parameters. On the basis of a highly symmetric "calibration sample", the point spread function of the microscope can be extracted and utilized for the correction of SPM images of unknowns that were recorded under essentially the same experimental conditions. A blunt scanning tunneling microscopy (STM) tip that consists of multiple mini-tips with electron orbital dimensions can be symmetrized on the basis of prior knowledge on the plane symmetry of a $2 \mathrm{D}$ periodic array $[3,4]$.

The removal of the effects of the point spread function of the microscope from the experimental data and the symmetrization of blunt STM tips both work because crystallographic image processing finds for each user selected plane group the positions in Fourier space about which the respective set of symmetries is the least broken. Then it declares these positions as the plane group origin and symmetrizes the Fourier transform coefficients of the image intensity to this origin by averaging over all symmetry equivalent coefficients. A Fourier transform of the symmetrized Fourier coefficients back into direct space leads subsequently to the symmetry enforced SPM image with all geometric distortions removed; see right hand sides of Figs. 1 and 2. The set of ratios of the experimental to the symmetry averaged Fourier coefficients contains useful information on the prevalent point spread function. "Essentially independent" STM images that a blunt scanning probe tip recorded are all symmetrized (and shifted) to the same crystallographic origin. A beneficial byproduct is then the enhancement of the signal to noise ratio, see right hand side of Fig. 1. Interference effects between the tunneling currents of the individual mini-tips (that make up the blunt tip) are symmetrized as well. Our preliminary analyses show, however, that systematic errors that may be associated with such interference effects are typically small.

The enforcing of plane symmetries is demonstrated below on STM images of fluorinated cobalt phthalocyanine on graphite (0001), refs. [3,4], Fig. 1, and hexaazatriphenylene-hexacarbonitrile on silver (111), ref. [5], Fig. 2. A dedicated crystallographic image processing program [6] is being 
developed by us because we think that the most popular crystallographic image processing program of the electron crystallography community [2] is not optimally suited to the processing of SPM images (as its main purpose is the extraction of the phases of structure factors as part of a suite [1]). The 80 layer groups (i.e. the 2D periodic 3D space symmetry groups, of which the 17 plane groups are subgroups) are the basis of the extension of "classical" crystallographic image processing (as described and implemented in refs. $[1,2]$ ) to images that were recorded with spin-polarized STMs or non-traditional SPMs (which are also often referred to as critical dimension SPMs). Future versions of our dedicated crystallographic image processing software [6] will be able to utilize these layer groups [7].

\section{References}

[1] X. Zou and S. Hovmöller, In: Industrial Applications of Electron Microscopy, Marcel Dekker Inc., 2003, pp. 583-614.

[2] S. Hovmöller, Ultramicroscopy 41 (1992) 121.

[3] P. Moeck et al., Proc. NSTI 2009 Vol. I (2009) 314, ISBN: 978-1-4398-1782-7.

[4] P. Moeck et al., AIP Conf. Proc. 1173 (2009) 294, ISBN: 978-0-7354-0712-1.

[5] H. Glowatzki et al., Nano Letters 8 (2008) 3825.

[6] P. Plachinda et al., Special Issue on "Interfacing Methodologies, Device Concepts and Architectural Strategies for Nanoscale Sensors and Systems" of the IEEE Transactions on Nanotechnology, submitted.

[7] This research was supported by a grant from Portland State University's Venture Fund.
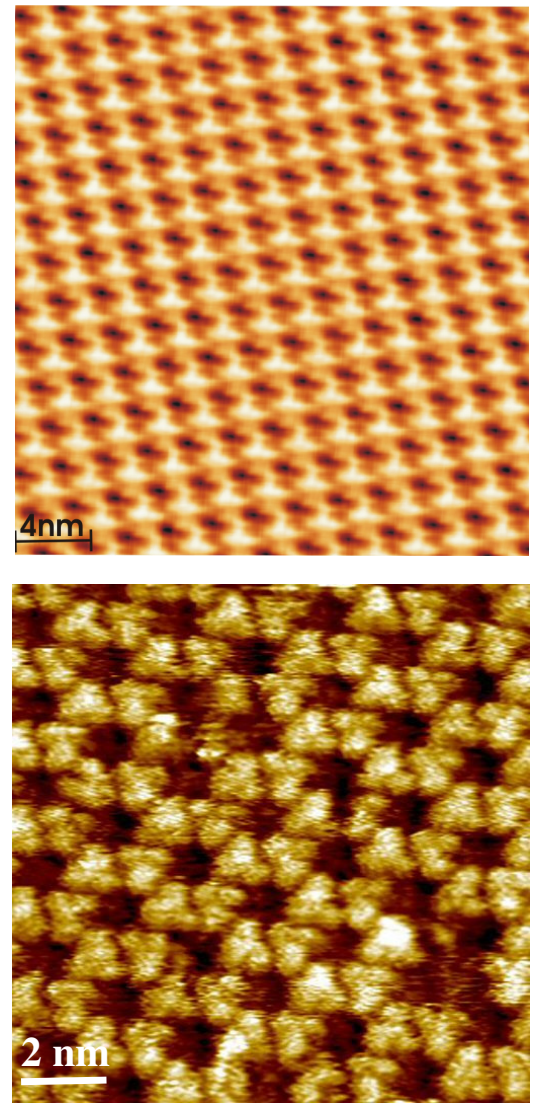

FIG. 1. STM images of fluorinated cobalt phthalocyanine molecules on graphite (0001) before (left) and after (right) crystallographic image processing. The blunt STM tip has been symmetrized to point group $4 \mathrm{~mm}$. A sketch of one molecule is superimposed on the $\mathrm{p} 4 \mathrm{~mm}$ symmetrized version of the image (right). We assume that its $2 \mathrm{D}$ point symmetry $(4 \mathrm{~mm})$ is not broken.

FIG. 2. STM images of hexaazatriphenylene-hexacarbonitrile molecules on silver (111) before (left) and after (right) crystallographic image processing. Our preliminary study suggests that the three mirror lines of the $2 \mathrm{D}$ point symmetry $(3 \mathrm{~mm})$ of an isolated molecule (below-right) are broken when the array is formed.

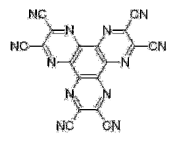

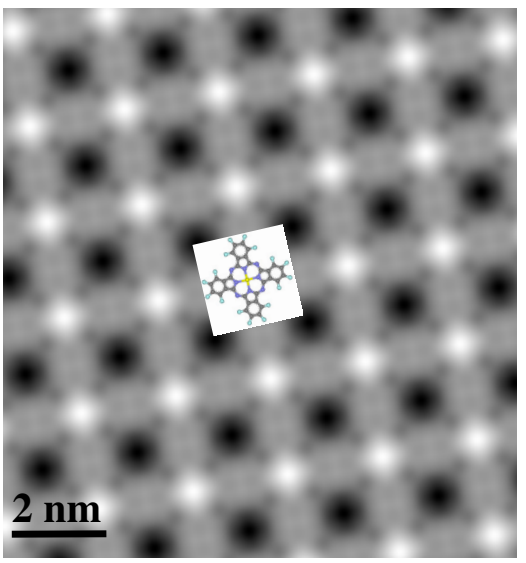

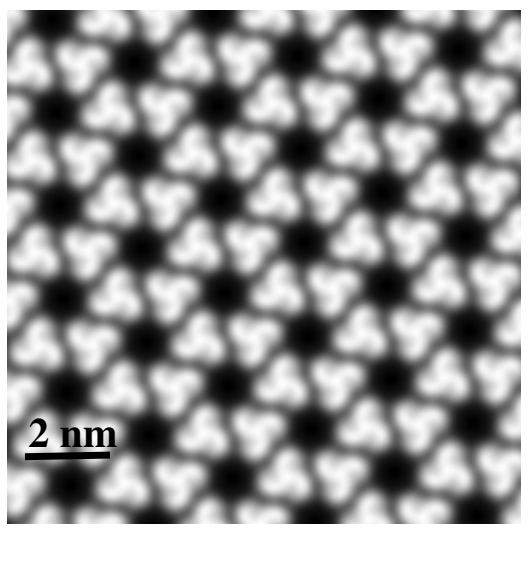

